\title{
Evaluation of multi-scale mineralized collagen-polycaprolactone composites for bone tissue engineering
}

\author{
D.W. Weisgerber ${ }^{1}$, K. Erning ${ }^{2}$, C.L. Flanagan ${ }^{3}$, S.J. Hollister ${ }^{3,4}$, B.A.C. Harley ${ }^{2,5}$ \\ ${ }^{1}$ Dept. of Material Science and Engineering \\ 2 Dept. of Chemical and Biomolecular Engineering \\ ${ }^{5}$ Carl R. Woese Institute for Genomic Biology \\ University of Illinois at Urbana-Champaign \\ Urbana, IL 61801 \\ ${ }^{3}$ Dept. of Biomedical Engineering \\ ${ }^{4}$ Dept. of Mechanical Engineering \\ University of Michigan \\ Ann Arbor, MI 48109
}

\author{
Corresponding Author: \\ B.A.C. Harley \\ Dept. of Chemical and Biomolecular Engineering \\ Carl R. Woese Institute for Genomic Biology \\ University of Illinois at Urbana-Champaign \\ 110 Roger Adams Laboratory \\ 600 S. Mathews Ave. \\ Urbana, IL 61801 \\ Phone: (217) 244-7112 \\ Fax: (217) 333-5052 \\ e-mail: bharley@illinois.edu
}

Keywords: collagen; polycaprolactone; biomaterial; multi-scale; craniofacial; bone 


\begin{abstract}
A particular challenge in biomaterial development for treating orthopedic injuries stems from the need to balance bioactive design criteria with the mechanical and geometric constraints governed by the physiological wound environment. Such trade-offs are of particular importance in large craniofacial bone defects which arise from both acute trauma and chronic conditions. Ongoing efforts in our laboratory have demonstrated a mineralized collagen biomaterial that can promote human mesenchymal stem cell osteogenesis in the absence of osteogenic media but that possesses suboptimal mechanical properties in regards to use in loaded wound sites. Here we demonstrate a multi-scale composite consisting of a highly bioactive mineralized collagenglycosaminoglycan scaffold with micron-scale porosity and a polycaprolactone support frame (PCL) with millimeter-scale porosity. Fabrication of the composite was performed by impregnating the PCL support frame with the mineral scaffold precursor suspension prior to lyophilization. Here we evaluate the mechanical properties, permeability, and bioactivity of the resulting composite. Results indicated that the PCL support frame dominates the bulk mechanical response of the composite resulting in a 6000-fold increase in modulus compared to the mineral scaffold alone. Similarly, the incorporation of the mineral scaffold matrix into the composite resulted in a higher specific surface area compared to the PCL frame alone. The increased specific surface area in the collagen-PCL composite promoted increased initial attachment of porcine adipose derived stem cells versus the PCL construct.
\end{abstract}




\section{Introduction}

Surgical intervention in large and geometrically complex craniofacial defects often necessitates either autologous or allogenic grafts to augment the natural wound healing process. Biomaterial approaches offer an alternative to the autologous implant with the potential to avoid donor site morbidity, alleviate concerns of allogenic supply and disease transmission, and allow real-time production of patient-specific implant geometries (Brydone et al., 2010). However, to promote physiological repair processes, such biomaterials must maintain their geometry and mechanical competence (Agrawal and Ray, 2001; Langer and Tirrell, 2004) particularly in the case of load bearing orthopedic injuries (Lipner et al., 2014; Wettergreen et al., 2005). Many injuries, including craniofacial defects, necessitate biomaterials capable of fitting non-trivial geometries to conform to an injury site, but also demonstrate mechanical stability under loading during implantation and routine physiological activity (Temple et al., 2014). In all cases, these biomaterials must remain bioactive supporting cellular infiltration, attachment, proliferation, and matrix synthesis.

Recently a range of biodegradable biomaterials have been described that promote pro-osteogenic processes within both the in vitro and in vivo environments. Here, the combination of inorganic and naturally derived polymers, such as in the case calcium phosphate mineralized chitosan biomaterials (Chen et al., 2015; Przekora and Ginalska, 2014; Przekora et al., 2014; Xie et al., 2015), have become a commonplace choice due to their ability to promote cellular osteogenic repair. While such biomaterials have demonstrated potential to promote osteogenic repair, concerns over ensuring geometric structure and mechanical competence under loading remain at the forefront. In that same spirit, previous and ongoing work within our group has evaluated the 
efficacy of a calcium phosphate mineralized collagen-glycosaminoglycan scaffold for the treatment of orthopedic injuries. Studies have highlighted the versatility in fabrication of these scaffolds with varied geometric, mechanical, and microstructural properties via freeze drying techniques (Caliari and Harley, 2011; Weisgerber et al., 2013). In vitro studies have likewise demonstrated the ability of the mineralized collagen-glycosaminoglycan scaffold to promote mesenchymal stem cell (MSC) osteogenic differentiation in the absence of osteogenic media or additional biomolecule supplementation (Weisgerber et al., 2015a). Interestingly, recent results suggest this mineralized collagen scaffold promotes MSC osteogenesis via activation of endogenous BMP pathways (Mobasseri et al., 2013; Ren et al., 2015). However, while this effort has demonstrated that the mineralized collagen-glycosaminoglycan matrix can promote proosteogenic activities, it has also highlighted the need for identifying strategies to increase the mechanical competence of these implants to meet the needs of in vivo applications (Weisgerber et al., 2013). And while increasing the relative density of these scaffolds can increase strength, it does so at the cost of reduced nutrient transport and cell penetration within the scaffold (O'Brien et al., 2007).

With a critical need in orthopedics for mechanically robust implants that can also be developed to fit patient and injury specific geometries, a variety of three-dimensional printing approaches have recently begun to garner significant attention. Early work by Wagoner Johnson et al., demonstrated the use of 3D-printed hydroxyapatite ceramic scaffolds able to enhance bone ingrowth and healing in vivo (Woodard et al., 2007). Ongoing efforts by Hollister et al. have demonstrated the use of polycaprolactone (PCL) constructs as well as additional control over incorporation of bioactive agents to enhancing biomolecule absorption and release (Patel et al., 
2015a; Suarez-Gonzalez et al., 2012), gene transfer (Hu et al., 2009), and resultant in vivo treatment of both cartilage and bone defects (Jeong et al., 2011; Patel et al., 2015b). The nature of the PCL construct allows rapid patient customization. After the 3D defect volume is computationally defined, a porous architecture can be designed by iterative addition of a welldefined unit cell structure to fill the desired shape (Hollister et al., 2005; Smith et al., 2007). Here a unit cell structure can be designed through an iterative loop process utilizing principles of mechanics, the deformation and failure modes to refine the unit cell design to meet the functional requirements (e.g., mechanical performance) of an implant into a specific injury site (Dias et al., 2012; Mitsak et al., 2011a). However, the fabrication process and biomechanical stability considerations often limit the porosity of these materials to pores/channels larger than 700 um (3.5mm dia. channels in current PCL constructs for a mandibular repair), resulting in low specific surface areas (surface area/volume) for cell attachment as well as pore sizes well above the $100-500 \mu \mathrm{m}$ range largely believed to be optimal for bone tissue engineering (Hulbert et al., 1970; Kuhne et al., 1994). So while 3D printed biomaterials offer mechanically robust, customizable implants, limits in printed feature size and low specific surface area represent critical dilemmas that must be addressed (Lipowiecki et al., 2014).

This manuscript describes development of a multi-scale biomaterial composite for the treatment of critically sized defects in bone that integrates mineralized collagen scaffolds with high osteogenic potential but suboptimal mechanical properties into 3D-printed PCL constructs with high strength. Multi-scale composites offer unique potential to satisfy multiple, disparate design criteria. Many mechanically efficient structures in nature (e.g., plant stems, porcupine quills, bones) employ multi-scale designs to satisfy mechanical and biological constraints (Karam and 
Gibson, 1994), suggesting such designs offer unique advantages for biomaterial design. Here we hypothesize that the combination of a bioactive collagen scaffold with micro-scale porosity with a strong PCL composite with mm-scale porosity will generate a composite with enhanced specific surface area and mechanical properties. We evaluate the average pore size, elastic modulus, permeability, and initial attachment of porcine adipose derived stem cells in multi-scale mineralized polycaprolactone-collagen-glycosaminoglycan (PCL-CGCaP) composite, comparing results to the individual polycaprolactone construct and the mineralized collagenglycosaminoglycan scaffold alone.

\section{Materials and Methods}

\subsection{Fabrication of polycaprolactone frames $(P C L)$.}

PCL frames were fabricated by selective laser sintering (SLS) of a powder precursor consisting of polycaprolactone and 4 wt\% hydroxyapatite (Dias et al., 2014; Hollister et al., 2015). The PCL support frames were $10 \mathrm{~mm}$ in height and $11 \mathrm{~mm}$ in diameter, the architecture of the frame was defined using a $67 \%$ porous $5 \mathrm{~mm}$ unit cell with $3.5 \mathrm{~mm}$ cylindrical pores through each axis

(Figure 1a). Specimen geometry was specified using a custom Interactive Data Language program (IDL, Research Systems, Inc.) with design criteria being stored as a .STL file for fabrication.

\subsection{Fabrication of mineralized scaffolds (CGCaP).}

CGCaP scaffolds were fabricated by lyophilization of a CGCaP precursor suspension as previously described (Harley et al., 2010; Weisgerber et al., 2013). Briefly, mineralized CGCaP precursor suspension was prepared by homogenizing $(1.9 \mathrm{w} / \mathrm{v} \%)$ bovine type I collagen (Collagen Matrix, Oakland, NJ) and (0.84 w/v \%) chondroitin-6-sulfate (Sigma-Aldrich, St. 
Louis, MO) with calcium salts $\left(\mathrm{Ca}(\mathrm{OH})_{2}, \mathrm{Ca}\left(\mathrm{NO}_{3}\right)_{2} \cdot 4 \mathrm{H}_{2} \mathrm{O}\right.$, Sigma-Aldrich, St. Louis, $\left.\mathrm{MO}\right)$ in phosphoric acid (Sigma-Aldrich, St. Louis, MO). This CGCaP precursor suspension was stored at $4^{\circ} \mathrm{C}$ and degassed prior to use. A Genesis freeze-dryer (VirTis, Gardener, NY) was used to lyophilize the precursor suspension in a custom 6 well (11.9 mm diameter, $10 \mathrm{~mm}$ height) polysulfone mold. Briefly, the lyophilization consisted of cooling from $20^{\circ} \mathrm{C}$ to $-10^{\circ} \mathrm{C}$ at a constant rate of $1^{\circ} \mathrm{C} / \mathrm{min}$ with a $2 \mathrm{~h}$ hold at $-10^{\circ} \mathrm{C}$ prior to sublimation at $0^{\circ} \mathrm{C}$ and $200 \mathrm{mTorr}$.

\subsection{Fabrication of polycaprolactone collagen scaffold composites (PCL-CGCaP).}

PCL-CGCaP composites were fabricated by first soaking the PCL frames in the CGCaP precursor suspension for 15 min. Excess CGCaP precursor suspension was removed from the PCL frames via blotting with Kim wipes (Kimberly-Clark, Roswell, GA). Following the degassing of both the wetted PCL frames and additional CGCaP precursor suspension the two were combined by placing the PCL frame into the previously described custom 6 well polysulfone mold (section 2.2) and injecting $1 \mathrm{ml} \mathrm{CGCaP} \mathrm{precursor} \mathrm{suspension} \mathrm{into} \mathrm{the} \mathrm{frame.}$ Composites were fabricated using an identical process conditions as described for creating CGCaP scaffold (section 2.2).

\subsection{Crosslinking and sterilization of specimens.}

All constructs used in the in vitro cell cultures underwent an ethanol sterilization with mineral scaffolds and composites undergoing additional cross-linking steps (Harley et al., 2007; Olde Damink et al., 1996). Briefly, all constructs sterilized by a $24 \mathrm{~h}$ soak in $100 \%$ ethanol overnight before being hydrated in sterile PBS for $24 \mathrm{~h}$. Scaffolds and composites were then soaked in a PBS solution of 1-ethyl-3-[3-dimethylaminopropyl] carbodiimide hydrochloride (EDAC, Sigma- 
Aldrich, St. Louis, MO) and N-hydroxysulfosuccinimide (NHS, Sigma-Aldrich, St. Louis, MO) at a molar ratio of 5:2:1 EADC:NHS: $\mathrm{COOH}$ where $\mathrm{COOH}$ represents the amount of carboxylic acid present in the collagen (Harley et al., 2007; Olde Damink et al., 1996).

\subsection{Pore size analysis of specimens.}

The visualization of the topology and interface between the PCL support and CGCaP scaffold within the composite was performed using SEM analysis with a JEOL JSM-6060LV Low Vacuum Scanning Electron Microscope (JEOL USA, Peabody, MA). To examine whether the addition of the PCL had any effect on the pore architecture within the composite, pore size analysis of samples was performed via the staining of collagen using aniline blue of samples embedded in a glycolmethacrylate (Polysciences Inc., Warrington, PA) (O'Brien et al., 2005; Weisgerber et al., 2013). Briefly, whole samples were divided into quarters and soaked in ethanol overnight prior to being stored in glycolmethacrylate monomer and catalyst benzoyl peroxide (Polysciences Inc., Warrington, PA) solution for $24 \mathrm{~h}$. Samples were then embedded in glycolmethacrylate in both longitudinal and transverse orientations and sectioned into $5 \mu \mathrm{m}$ slices. Slices were mounted, stained with aniline blue (Thermo Fisher Scientific Inc., Waltham, MA), and imaged with an optical microscope (Leica Microsystems, Wetzlar, Germany). Subsequent image analysis was performed using a linear intercept method using a custom Matlab program to determine average pore size (O'Brien et al., 2004; Pontius, 1998).

\subsection{Mechanical behavior of specimens.}

The stress-strain behavior of the PCL frame, CGCaP scaffold, and PCL-CGCaP composite was measured via compressive loading in a TA.XTplus Texture Analyzer (StableMicro Systems Ltd., 
Surrey, UK) using a $5 \mathrm{~kg}$ load cell. Samples were loaded to $4 \mathrm{~kg}$ or $50 \%$ strain at a rate of 0.01 $\mathrm{mm} / \mathrm{min}$. The elastic moduli of each sample (11 mm diameter, $10 \mathrm{~mm}$ height) was determined from the linear elastic region of each stress-strain plot. Specimens were tested as dry (room temperature and humidity following lyophilization) or as hydrated (wet; fully hydrated then submerged in $18 \mathrm{M} \Omega$-cm de-ionized water) as previously described (Harley et al., 2007). The compressive strength of the PCL support alone and the PCL-CGCaP composite was measured using an Instron 5943 mechanical tester (Instron, Norwood, MA) under dry conditions with a $100 \mathrm{~N}$ load cell. Briefly, samples were compressed to failure at a rate of $1 \mathrm{~mm} / \mathrm{min}$, with the compressive strength determined from the stress-strain plot using conventional analysis methods (Harley et al., 2007).

\subsection{Permeability of specimens.}

Permeability was measured as previously described (Lipowiecki et al., 2014; O'Brien et al., 2007; Weisgerber et al., 2013). Briefly, axial permeability was assessed with flow through using a custom polycarbonate rig and a constant pressure head $(\Delta P)$ of $26.7 \mathrm{~cm}$ of de-ionized water. The resulting flow rate $(Q)$ of de-ionized water with viscosity $(\mu)$ through a defined crosssectional area $(A)$ and thickness $(I)$ of each sample (empty chamber, PCL frame, CGCaP scaffold, and PCL-CGCaP composite, $\mathrm{n}=3$ ) was measured using a graduated cylinder (10 \pm 0.1 mL). Sample permeability (K) was defined via Darcy’s law (O'Brien et al., 2007):

$$
K=\frac{Q \cdot l \cdot \mu}{\Delta P \cdot A}
$$

2.8. Porcine adipose derived stem cell (pASC) culture and seeding. 
To examine the effect of the CGCaP mineral scaffold on composite bioactivity porcine adipose derived stem cells (gift of Dr. M. Wheeler, Department of Animal Science, UIUC, Urbana, IL) were seeded onto the PCL support and the composite. Porcine adipose derived stem cells were expanded at $37^{\circ} \mathrm{C}$ and $5 \% \mathrm{CO}_{2}$ in complete mesenchymal stem cell growth media (low glucose DMEM, 10\% MSC FBS and 1\% antibiotic-antimycotic). Passage 8 pASCs were seeded onto PCL frames and PCL-CGCaP composites using one of two different seeding methods. Briefly, a previously described static seeding method was used to seed a total of $1.0 \times 10^{6}$ cells onto each sample (Caliari and Harley, 2011). Alternatively, samples were soaked in a suspension of 1.0 $\mathrm{x} 10^{6}$ cells in complete mesenchymal stem cell growth media for a period of $2 \mathrm{~h}$. Subsequently, all pASC seeded samples were cultured at $37^{\circ} \mathrm{C}$ and $5 \% \mathrm{CO}_{2}$ in complete mesenchymal stem cell growth media for $24 \mathrm{~h}$.

\subsection{Measurement of porcine ASC metabolic activity.}

The metabolic activity of pASC seeded specimens was quantified via an alamarBlue ${ }^{\circledR}$ assay (Tierney et al., 2009). Briefly, after $24 \mathrm{~h}$ of culture, cell-seeded constructs were incubated in an alamarBlue ${ }^{\circledR}$ solution (Invitrogen) at $37^{\circ} \mathrm{C}$ under gentle shaking. The fluorescence of the reduced resazurin byproduct, resorufin, via the metabolically active pASCs was compared to a prepared standard. Using a F200 spectrophotometer (Tecan) the fluorescence was measured at 540(52)/580(20) nm (excitation/ emission). The resulting metabolic activity of each sample (n

=6) was normalized to the metabolic activity of a control constructed from the metabolic activity of the number of cells seeded $\left(1.0 \times 10^{6}\right.$ cells).

\subsection{Western blot analysis.}


Porcine ASCs were seeded in CGCaP scaffolds or CGCaP-PCL composites and maintained in culture for up to 7 days. Protein lysates were collected at days 4 and 7 using a combination of Phosphate Inhibitor Cocktail (Sigma-Aldrich, St. Louis, MO) and Phosphatase Inhibitor Cocktails 2 and 3 (Sigma-Aldrich, St. Louis, MO) in a RIPA lysis buffer. Total protein content was assessed using a Micro BCA Protein Assay Kit (Thermo Fisher Scientific, Waltham, MA). Western blot analysis was subsequently performed using $4 \mu$ g of protein lysate per lane, using primary antibodies against Smad1 and phosphorylated Smad1/5/8 (pSmad1/5/8), with $\beta$-actin as a loading control (all from Cell Signaling Technology, Danvers, MA) followed by a 1:5000 dilution of HRP-IgG (Cell Signaling Technology). Blots were imaged using a SuperSignal West Femto Maximum Sensitivity Substrate (Thermo Fisher Scientific, Waltham, MA) via an Image Quant LAS 4010 (GE Healthcare Life Sciences, Little Chalfont, United Kingdom).

\subsection{Statistics analysis.}

Statistical analysis was performed via analysis of variance (ANOVA) tests after which a TukeyHSD post-hoc test was used. Independent factors included the seeding method (static seeding vs cell soak) and scaffold type (PCL frame vs PCL-CGCaP composite). Mechanical and metabolic studies were carried out with $n=6$ samples per group, while permeability was carried out with $n$

$=3$ samples per group. Significance was set at $p<0.05$. Error bars are reported as standard deviation unless otherwise stated.

\section{Results}

\subsection{Effect of PCL support on microstructural features of the CG scaffold compartment.}


A qualitative analysis of the microstructural differences observed via SEM did not identify any apparent differences between the mineralized CGCaP matrix and PCL support within the PCLCGCaP composite and the CGCaP scaffold and PCL support construct alone (Figure 1c). The average pore size of the CGCaP scaffold and collagen-glycosaminoglycan matrix within the PCL-CGCaP composite was evaluated using aniline blue staining of glycolmethacrylate embedded samples (Figure 2). No significant difference between groups was observed.

3.2. Effect of incorporating a PCL support on the mechanical behavior of collagen scaffolds. Mechanical analysis of the PCL frame, CGCaP scaffold, and the PCL-CGCaP composite was tested in compression (Figure 3). A significant $(\mathrm{p}<0.05)$ difference in the elastic modulus was observed between the PCL-CGCaP composite and the mineralized CGCaP scaffold. Not surprisingly, no significant difference in elastic modulus was observed between the PCL frame and the PCL-CGCaP composite. The compressive strength of the PCL-CGCaP composite (762.0 $\pm 70.6 \mathrm{kPa})$ was significantly $(p<0.05)$ that that for the PCL support alone $(690.8 \pm 63.6 \mathrm{kPa})$. Failure of the specimens occurred symmetrically at the edges of the PCL unit cells.

\subsection{Comparison of the permeability of the PCL support and PCL-CGCaP composite.}

The permeability of the PCL frame and the PCL-CGCaP composite was measured and compared to the permeability of the mineralized CGCaP scaffold (Figure 4). A significant $(\mathrm{p}<0.05)$ difference in permeability between the PCL frame and the PCL-CGCaP composite was observed. In contrast, the permeability of the mineralized CGCaP scaffold was 1.05 times the PCL-CGCaP composite, a non-significant ( $\mathrm{p}>0.8$ ) difference. In order to ensure that the permeability of the PCL frame was not limited by the experimental apparatus, the permeability 
of the empty permeability chamber was measured to be 1.4 times higher, a significant $(\mathrm{p}<0.05)$ difference from the PCL frame.

\subsection{Measurement of porcine ASC cell attachment via metabolic activity.}

The metabolic activity of the PCL frame and the PCL-CGCaP composite was determined after $24 \mathrm{~h}$ as proxy for initial cell attachment and resultant metabolic health (Figure 5a). A significant $(p<0.05)$ increase in metabolic activity was observed in the higher surface area per volume PCL-CGCaP composite compared to the smaller degree of surface area per volume of the PCL alone. Regardless of the use of two different cell seeding methods (static seeding vs cell soak), a significant $(\mathrm{p}<0.05)$ increase in metabolic activity was measured for the PCL-CGCaP composite versus the PCL support alone.

\subsection{Monitoring scaffold-induced osteogenic activity via pSmad1/5/8 expression.}

Previous investigations into mechanism underlying stem cell osteogenic differentiation in the CGCaP scaffolds have identified early (within 7 day) activation of BMP receptor pathways and upregulation of Smad1/5/8 phosphorylation in the CGCaP scaffold as key markers of osteogenesis (Lee et al., 2015; Ren et al., 2015; Ren et al., 2016). To confirm that inclusion of the PCL cage into the CGCaP scaffold to form the composite did not negatively impact the osteogenic potential of the scaffold, we examined Smad1 and pSmad1/5/8 via Western blot after 4 and 7 days of in vitro culture. Here, we compared results for the pASC seeded CGCaP scaffold alone versus the pASC seeded PCL-CGCaP composite. Bands associated with Smad1 (60 kDa) and $\beta$-actin (45 kDa) were clearly identified. Critically, increasing expression of pSmad1/5/8 (60 $\mathrm{kDa}$ ) was detected between days 4 and 7 in both constructs (Figure 5b). Importantly, no 
reduction in pSmad1/5/8 were observed between in the PCL-CGCaP composite versus the CGCaP scaffold alone.

\section{Discussion}

This study demonstrates a generalizable approach, based on multi-scale composite concepts, to generate a PCL-CGCaP composite biomaterial for applications in craniofacial repair. First, a versatile SLS approach is used to generate a 3D PCL support construct that can be subsequently filled with mineralized collagen-glycosaminoglycan suspensions then lyophilized to generate an arbitrary composite geometry. Previous efforts have also demonstrated the potential for patient customization of PCL implants as well as the use of custom-printed PCL sleeves as well as standard bone plates to secure the PCL implant and stabilize the wound site immediately following surgical intervention (Dias et al., 2014; Hollister et al., 2015; Mitsak et al., 2011b; Williams et al., 2005; Zopf et al., 2014). Here we hypothesize that by combining the PCL frame and mineralized CGCaP scaffold into the PCL-CGCaP composite the most favorable properties, increased elastic modulus and increased surface area, will be maintained for future in vivo studies. The impact of incorporating the mineralized CGCaP scaffold into the PCL frame, creating the PCL-CGCaP composite, was determined by measurements of the pore size, mechanics, microstructure, and cell attachment.

In support of our hypothesis, we identified that the incorporation of the mineral CGCaP scaffold into the PCL frame significantly increased the bulk modulus of the material compared to the mineral CGCaP scaffold alone (Figure 3). This drastic 6000-fold increase in modulus to $6.8 \pm$ 0.4 MPa approaches the range necessary to mimic in vivo cancellous bone (2 MPa to $14.8 \mathrm{GPa}$ ) 
(Choi et al., 1990; Hannink and Arts, 2011; Rho et al., 1993). This change is due to the incorporation of the PCL frame acting as the load bearing component. Notably, the increase in mechanical properties in the collagen-PCL composite was achieved without any deleterious changes to the average pore size of the collagen scaffold itself (Figure 2). Here, significant decreases in pore size or increased in scaffold density would have significantly impacted specific surface area and nutrient biotransport, which would compromise the bioactivity of the composite (Harley and Gibson, 2008; O'Brien et al., 2007). Previous work has validated the use of cellular solids theory calculations to predict changes in scaffold modulus dependent on changes in scaffold relative density squared (O'Brien et al., 2005), suggesting the 6000-fold increase in modulus accomplished via our PCL composite approach would require a greater than 70-fold increase in scaffold density in a non-composite design, rendering the scaffold poorly suited for cell infiltration or biotransport of oxygen and nutrients. It is further anticipated that this composite architecture will protect the CGCaP matrix within the PCL-CGCaP composite from loads exceeding its yield point, allowing the CGCaP matrix to be tailored specifically to meet requirements regarding cell bioactivity. Previous work has already demonstrated the bioactivity of this matrix and its effect on endogenous BMP2 production (Ren et al., 2015), integrin activation (Caliari et al., 2015c), mechanotransduction (Caliari and Harley, 2014), and gene expression (Weisgerber et al., 2015a). So removing the requirement for meeting mechanical constraints offers the potential to further optimize the scaffold solely in the context of driving enhanced MSC osteogenic activity. This finding also suggests a more generic approach to overcome trade-offs between mechanical robustness and qualities associated with bioactivity via composite design principles (Bonfield, 1988; Hannink and Arts, 2011; Kurtz and Devine, 2007). An additional analysis of the compressive strength of the PCL support versus the PCL-CGCaP 
composite identified a significant increase in the compressive strength of the composite, likely due to the increased density of the PCL-CGCaP composite as a result of the inclusion of the scaffold within the PCL cage.

We then examined the effect of the specific surface area of the constructs on construct permeability. In the case of the CGCaP scaffold and PCL-CGCaP composite, a high degree of specific surface area was reflected in the significantly lower permeability compared to the PCL support alone (Figure 4). The relatively lower specific surface area and resultant increased permeability in the 3D printed PCL construct has been previously attributed to limitations in feature size (mm-scale, rather than micron-scale, pores) (Lipowiecki et al., 2014). The significance of specific surface area as a biomaterial design parameter has similarly been addressed (Harley and Gibson, 2008; O'Brien et al., 2007; O'Brien et al., 2005). If too low, the specific surface area can severely limit attachment and alter fluid flow within a construct (Jungreuthmayer et al., 2009), while if too high results in poor infiltration into the construct. Earlier efforts regarding development of collagen-glycosaminoglycan scaffolds have examined the influence in changes in scaffold specific surface area on a range of cellular responses (O'Brien et al., 2007). Importantly these results again suggest it is possible to focus design of the collagen scaffold compartment on structural and bioactivity, rather than mechanical, concerns.

The influence of specific surface area on cell response was then demonstrated via comparison of early metabolic activity metrics for porcine ASCs seeded within the PCL support versus the PCL-CGCaP composite. Here the metabolic activity of pASCs within the PCL support alone was significantly $(\mathrm{p}<0.05)$ lower than that within the PCL-CGCaP composite at 24 hours regardless 
of seeding method tested (Figure 5a). This observed difference in metabolic activity is likely a function of an increase in initial cell infiltration and attachment as a result of the enhanced specific surface area of the composite. Here the 24 hour timepoint was used both to be consistent with previous work that evaluated the effect of scaffold specific surface area on cell attachment (O'Brien et al., 2005), but also because 24 hours offers only a limited period for any potential proliferation which could affect the results. Interestingly, the static seeding method led to a significant increase in cell attachment compared to the cell soak method in the presence of the increased specific surface area of the PCL-CGCaP composite, suggesting a preferred cell seeding method for future in vitro and in vivo experiments.

We subsequently examined whether the PCL component of the PCL-CGCaP composite altered the previously demonstrated osteogenic capacity of the CGCaP scaffold (Lee et al., 2015; Ren et al., 2015; Ren et al., 2016; Weisgerber et al., 2015b). As early (within 7 days) upregulation of Smad1/5/8 phosphorylation was observed as a key marker of enhanced osteogenesis in the CGCaP scaffold versus non-mineralized scaffold variants (Lee et al., 2015; Ren et al., 2015; Ren et al., 2016), we compared pASC Smad1/5/8 phosphorylation in the CGCaP scaffold alone versus the PCL-CGCaP composite. No loss in pSmad1/5/8 was observed in the PCL-CGCaP composite versus the CGCaP scaffold alone at either 4 or 7 days (Figure 5b), suggesting the CGCaP scaffold within the composite retains its osteogenic potential. Long-term in vivo assays are currently ongoing to assess the quality of new bone formation in the composite.

Additionally, incorporation of the mineralized collagen scaffold into the PCL frame also provides increased surface area for generalized growth factor immobilization along with the 
possibility of differential factor immobilization and sequential vs. simultaneous release. Previous work within our laboratories and within the literature has demonstrated growth factor immobilization and release strategies within collagen scaffolds (Alsop et al., 2014; Caliari et al., 2015a; Caliari et al., 2015b; Martin et al., 2011; Shen et al., 2008), on polycaprolactone constructs (Zhang et al., 2010), as well as other collagen-coated polycaprolactone biomaterial constructs (Singh et al., 2012). The multi-scale composite approach presented here has the exciting potential for orthogonal immobilization and release of multiple biomolecular signals from distinct composite components.

\section{Conclusions}

This work described the development of a multi-scale composite approach for engineering biomaterials for critical-size bone defects. This approach was intended to circumvent the typical design trade-offs found in biomaterials where there is often a tension between optimizing the bioactivity versus mechanical strength of an implant. We report a strategy to create a PCLCGCaP composite that combines a CGCaP scaffold able to promote MSC osteogenesis in the absence of traditional osteogenic supplementation with a mechanically-robust, patientcustomizable PCL support frame generated via 3D-printing. The PCL-CGCaP composite demonstrated a bulk modulus significantly higher than the CGCaP scaffold alone but consistent with the PCL support alone. Similarly, the permeability of the PCL-CGCaP composite was dictated by the CGCaP scaffold compartment. Evaluation of porcine ASC seeded constructs revealed that the increased specific surface area of the composite led to significantly higher overall metabolic activity after 24h consistent with higher ASC attachment rates. In conclusion, the PCL-CGCaP composite was found to be mechanically robust compared to the CGCaP 
scaffold while maintaining the inherent bioactivity of the CGCaP scaffold and a degree of customizability inherent in its multi-scale composite design.

\section{Acknowledgements}

The authors would like to acknowledge the generous donation of porcine adipose derived stem cells isolated and contributed by Dr. Mathew Wheeler (Department of Animal Science, UIUC, Urbana, IL), and Karen Doty (Veterinary Sciences, UIUC) for sectioning of GMA embedded samples. Project S-14-54H was supported by the AO Foundation (Switzerland). This research was carried out in part in the Frederick Seitz Materials Research Laboratory Central Facilities, University of Illinois, which are partially supported by the U.S. Department of Energy under grants DE-FG02-07ER46453 and DE-FG02-07ER46471. 


\section{Figure Captions}

Figure 1. (a) A visual representation and an image of the mineral CGCaP scaffold, the PCL support, and the CGCaP-PCL composite respectively. (b) Representative SEM images of the CGCaP scaffold and PCL support alone (top). Representative SEM images taken within the collagen-PCL composite of the CGCaP matrix zone (bottom left), the interface between the CGCaP matrix and the PCL support (dashed line; bottom middle), and the PCL support alone (bottom right). Scale bar: $100 \mu \mathrm{m}$.

Figure 2. The average pore size within the CGCaP scaffold compared to the average pore size of the mineralized collagen-GAG component within the CGCaP-PCL composite. NS: no significance between the two groups.

Figure 3. The elastic modulus of the CGCaP scaffold, PCL support, and CGCaP-PCL composite as measured by both dry and hydrated unconfined compression. *: significance $(p<0.05)$ between indicated groups.

Figure 4. Permeability of the empty permeability chamber, PCL support, CGCaP scaffold, and CGCaP-PCL composite respectively as measured by a constant head permeability test. *: significant $(\mathrm{p}<0.05)$ difference between indicated groups.

Figure 5. (a) Initial cell attachment as measured by AlamarBlue between the PCL support and the CGCaP-PCL composite using two cell seeding methods, static seeding and a 2 h cell soak. *: significance ( $<<0.05$ ) between indicated groups. (b) Analysis of expression levels of total Smad1, phosphorylated Smad1/5/8 (pSmad1/5/8), and $\beta$-actin via Western blot for pASCs cultured in CGCaP scaffold alone (positive control) or the PCL-CGCaP composite for 4 or 7 days. 


\section{References}

Agrawal, C.M., Ray, R.B., 2001. Biodegradable polymeric scaffolds for musculoskeletal tissue engineering. J Biomed Mater Res 55, 141-150.

Alsop, A.T., Pence, J.C., Weisgerber, D.W., Harley, B.A., Bailey, R.C., 2014. Photopatterning of vascular endothelial growth factor within collagen-glycosaminoglycan scaffolds can induce a spatially confined response in human umbilical vein endothelial cells. Acta Biomater 10, 47154722.

Bonfield, W., 1988. Composites for bone replacement. J Biomed Eng 10, 522-526.

Brydone, A.S., Meek, D., Maclaine, S., 2010. Bone grafting, orthopaedic biomaterials, and the clinical need for bone engineering. Proc Inst Mech Eng H 224, 1329-1343.

Caliari, S.R., Gonnerman, E.A., Grier, W.K., Weisgerber, D.W., Banks, J.M., Alsop, A.J., Lee, J.-S., Bailey, R.C., Harley, B.A.C., 2015a. Collagen scaffold arrays for combinatorial screening of biophysical and biochemical regulators of cell behavior. Advanced healthcare materials 4, 5864.

Caliari, S.R., Grier, W.K., Weisgerber, D.W., Mahmassani, Z., Boppart, M.D., Harley, B.A.C., 2015b. Collagen scaffolds incorporating coincident gradations of instructive structural and biochemical cues for osteotendinous junction engineering. Advanced healthcare materials 4, 831837.

Caliari, S.R., Harley, B.A., 2011. The effect of anisotropic collagen-GAG scaffolds and growth factor supplementation on tendon cell recruitment, alignment, and metabolic activity.

Biomaterials 32, 5330-5340.

Caliari, S.R., Harley, B.A., 2014. Collagen-GAG scaffold biophysical properties bias MSC lineage choice in the presence of mixed soluble signals. Tissue Eng Part A 20, 2463-2472. Caliari, S.R., Weisgerber, D.W., Grier, W.K., Mahmassani, Z., Boppart, M.D., Harley, B.A., 2015c. Collagen Scaffolds Incorporating Coincident Gradations of Instructive Structural and Biochemical Cues for Osteotendinous Junction Engineering. Adv Healthc Mater 4, 831-837. Chen, L., Li, B., Xiao, X., Meng, Q., Li, W., Yu, Q., Bi, J., Cheng, Y., Qu, Z., 2015. Preparation and evaluation of an Arg-Gly-Asp-modified chitosan/hydroxyapatite scaffold for application in bone tissue engineering. Mol Med Rep.

Choi, K., Kuhn, J.L., Ciarelli, M.J., Goldstein, S.A., 1990. The elastic moduli of human subchondral, trabecular, and cortical bone tissue and the size-dependency of cortical bone modulus. J Biomech 23, 1103-1113.

Dias, M.R., Fernandes, P.R., Guedes, J.M., Hollister, S.J., 2012. Permeability analysis of scaffolds for bone tissue engineering. J Biomech 45, 938-944.

Dias, M.R., Guedes, J.M., Flanagan, C.L., Hollister, S.J., Fernandes, P.R., 2014. Optimization of scaffold design for bone tissue engineering: A computational and experimental study. Medical engineering \& physics 36, 448-457.

Hannink, G., Arts, J.J.C., 2011. Bioresorbability, porosity and mechanical strength of bone substitutes: What is optimal for bone regeneration? Injury-International Journal of the Care of the Injured 42, S22-S25.

Harley, B.A., Leung, J.H., Silva, E.C., Gibson, L.J., 2007. Mechanical characterization of collagen-glycosaminoglycan scaffolds. Acta Biomater 3, 463-474.

Harley, B.A., Lynn, A.K., Wissner-Gross, Z., Bonfield, W., Yannas, I.V., Gibson, L.J., 2010. Design of a multiphase osteochondral scaffold. II. Fabrication of a mineralized collagenglycosaminoglycan scaffold. J Biomed Mater Res A 92, 1066-1077. 
Harley, B.A.C., Gibson, L.J., 2008. In vivo and in vitro applications of collagen-GAG scaffolds. Chem Eng J 137, 102-121.

Hollister, S.J., Flanagan, C.L., Zopf, D.A., Morrison, R.J., Nasser, H., Patel, J.J., Ebramzadeh, E., Sangiorgio, S.N., Wheeler, M.B., Green, G.E., 2015. Design control for clinical translation of 3D printed modular scaffolds. Annals of biomedical engineering 43, 774-786.

Hollister, S.J., Lin, C.Y., Saito, E., Lin, C.Y., Schek, R.D., Taboas, J.M., Williams, J.M., Partee, B., Flanagan, C.L., Diggs, A., Wilke, E.N., Van Lenthe, G.H., Muller, R., Wirtz, T., Das, S., Feinberg, S.E., Krebsbach, P.H., 2005. Engineering craniofacial scaffolds. Orthod Craniofac Res 8, 162-173.

Hu, W.W., Elkasabi, Y., Chen, H.Y., Zhang, Y., Lahann, J., Hollister, S.J., Krebsbach, P.H., 2009. The use of reactive polymer coatings to facilitate gene delivery from poly (epsiloncaprolactone) scaffolds. Biomaterials 30, 5785-5792.

Hulbert, S.F., Young, F.A., Mathews, R.S., Klawitter, J.J., Talbert, C.D., Stelling, F.H., 1970. Potential of ceramic materials as permanently implantable skeletal prostheses. J Biomed Mater Res 4, 433-456.

Jeong, C.G., Zhang, H., Hollister, S.J., 2011. Three-dimensional poly(1,8-octanediol-co-citrate) scaffold pore shape and permeability effects on sub-cutaneous in vivo chondrogenesis using primary chondrocytes. Acta Biomater 7, 505-514.

Jungreuthmayer, C., Donahue, S.W., Jaasma, M.J., Al-Munajjed, A.A., Zanghellini, J., Kelly, D.J., O'Brien, F.J., 2009. A comparative study of shear stresses in collagen-glycosaminoglycan and calcium phosphate scaffolds in bone tissue-engineering bioreactors. Tissue Eng Part A 15, 1141-1149.

Karam, G.N., Gibson, L.J., 1994. Biomimicking of animal quills and plant stems - natural cylindrical-shells with foam cores. Mater. Sci. Eng. C Biomim. Mater. Sens. Syst. 2, 113-132. Kuhne, J.H., Bartl, R., Frisch, B., Hammer, C., Jansson, V., Zimmer, M., 1994. Bone formation in coralline hydroxyapatite. Effects of pore size studied in rabbits. Acta Orthop Scand 65, 246252.

Kurtz, S.M., Devine, J.N., 2007. PEEK biomaterials in trauma, orthopedic, and spinal implants. Biomaterials 28, 4845-4869.

Langer, R., Tirrell, D.A., 2004. Designing materials for biology and medicine. Nature 428, 487492.

Lee, J.C., Pereira, C.T., Ren, X., Huang, W., Weisgerber, D.W., Yamaguchi, D.T., Harley, B.A.C., Miller, T.A., 2015. Optimizing collagen scaffolds for bone engineering: effects of crosslinking and mineral content on structural contraction and osteogenesis. J Craniofac Surg 26, 1992-1996.

Lipner, J., Liu, W., Liu, Y., Boyle, J., Genin, G.M., Xia, Y., Thomopoulos, S., 2014. The mechanics of PLGA nanofiber scaffolds with biomimetic gradients in mineral for tendon-to-bone repair. J Mech Behav Biomed Mater 40, 59-68.

Lipowiecki, M., Ryvolova, M., Tottosi, A., Kolmer, N., Naher, S., Brennan, S.A., Vazquez, M., Brabazon, D., 2014. Permeability of rapid prototyped artificial bone scaffold structures. J

Biomed Mater Res A 102, 4127-4135.

Martin, T.A., Caliari, S.R., Williford, P.D., Harley, B.A., Bailey, R.C., 2011. The generation of biomolecular patterns in highly porous collagen-GAG scaffolds using direct photolithography. Biomaterials 32, 3949-3957.

Mitsak, A.G., Kemppainen, J.M., Harris, M.T., Hollister, S.J., 2011a. Effect of polycaprolactone scaffold permeability on bone regeneration in vivo. Tissue Eng Part A 17, 1831-1839. 
Mitsak, A.G., Kemppainen, J.M., Harris, M.T., Hollister, S.J., 2011b. Effect of polycaprolactone scaffold permeability on bone regeneration in vivo. Tissue Eng Part A 17, 1831-1839.

Mobasseri, S.A., Terenghi, G., Downes, S., 2013. Micro-structural geometry of thin films intended for the inner lumen of nerve conduits affects nerve repair. J Mater Sci Mater Med 24, 1639-1647.

O'Brien, F.J., Harley, B.A., Waller, M.A., Yannas, I.V., Gibson, L.J., Prendergast, P.J., 2007. The effect of pore size on permeability and cell attachment in collagen scaffolds for tissue engineering. Technol Health Care 15, 3-17.

O'Brien, F.J., Harley, B.A., Yannas, I.V., Gibson, L., 2004. Influence of freezing rate on pore structure in freeze-dried collagen-GAG scaffolds. Biomaterials 25, 1077-1086.

O'Brien, F.J., Harley, B.A., Yannas, I.V., Gibson, L.J., 2005. The effect of pore size on cell adhesion in collagen-GAG scaffolds. Biomaterials 26, 433-441.

Olde Damink, L.H., Dijkstra, P.J., van Luyn, M.J., van Wachem, P.B., Nieuwenhuis, P., Feijen, J., 1996. Cross-linking of dermal sheep collagen using a water-soluble carbodiimide.

Biomaterials 17, 765-773.

Patel, J.J., Flanagan, C.L., Hollister, S.J., 2015a. Bone Morphogenetic Protein-2 Adsorption onto Poly-varepsilon-caprolactone Better Preserves Bioactivity In Vitro and Produces More Bone In Vivo than Conjugation Under Clinically Relevant Loading Scenarios. Tissue Eng Part C Methods 21, 489-498.

Patel, J.J., Modes, J.E., Flanagan, C.L., Krebsbach, P.H., Edwards, S.P., Hollister, S.J., 2015b. Dual Delivery of EPO and BMP2 from a Novel Modular Poly-varepsilon-Caprolactone Construct to Increase the Bone Formation in Prefabricated Bone Flaps. Tissue Eng Part C Methods.

Pontius, J.S., 1998. Estimation of the mean in line intercept sampling. Environ Ecol Stat 5, 371379.

Przekora, A., Ginalska, G., 2014. Addition of 1,3-beta-D-glucan to chitosan-based composites enhances osteoblast adhesion, growth, and proliferation. Int J Biol Macromol 70, 474-481. Przekora, A., Palka, K., Ginalska, G., 2014. Chitosan/beta-1,3-glucan/calcium phosphate ceramics composites--novel cell scaffolds for bone tissue engineering application. J Biotechnol 182-183, 46-53.

Ren, X., Bischoff, D., Weisgerber, D.W., Lewis, M.S., Tu, V., Yamaguchi, D.T., Miller, T.A., Harley, B.A., Lee, J.C., 2015. Osteogenesis on nanoparticulate mineralized collagen scaffolds via autogenous activation of the canonical BMP receptor signaling pathway. Biomaterials 50, 107-114.

Ren, X., Tu, V., Bischoff, D., Weisgerber, D.W., Lewis, M.S., Yamaguchi, D.T., Miller, T.A., Harley, B.A.C., Lee, J.C., 2016. Nanoparticulate mineralized collagen scaffolds induce in vivo bone regeneration independent of progenitor cell loading or exogenous growth factor stimulation. Biomaterials.

Rho, J.Y., Ashman, R.B., Turner, C.H., 1993. Young's modulus of trabecular and cortical bone material: ultrasonic and microtensile measurements. J Biomech 26, 111-119.

Shen, Y.H., Shoichet, M.S., Radisic, M., 2008. Vascular endothelial growth factor immobilized in collagen scaffold promotes penetration and proliferation of endothelial cells. Acta Biomater 4, 477-489.

Singh, S., Wu, B.M., Dunn, J.C., 2012. Delivery of VEGF using collagen-coated polycaprolactone scaffolds stimulates angiogenesis. J Biomed Mater Res A 100, 720-727. 
Smith, M.H., Flanagan, C.L., Kemppainen, J.M., Sack, J.A., Chung, H., Das, S., Hollister, S.J., Feinberg, S.E., 2007. Computed tomography-based tissue-engineered scaffolds in craniomaxillofacial surgery. Int J Med Robot 3, 207-216.

Suarez-Gonzalez, D., Barnhart, K., Migneco, F., Flanagan, C., Hollister, S.J., Murphy, W.L., 2012. Controllable mineral coatings on PCL scaffolds as carriers for growth factor release. Biomaterials 33, 713-721.

Temple, J.P., Hutton, D.L., Hung, B.P., Huri, P.Y., Cook, C.A., Kondragunta, R., Jia, X., Grayson, W.L., 2014. Engineering anatomically shaped vascularized bone grafts with hASCs and 3D-printed PCL scaffolds. J Biomed Mater Res A 102, 4317-4325.

Tierney, C.M., Jaasma, M.J., O'Brien, F.J., 2009. Osteoblast activity on collagen-GAG scaffolds is affected by collagen and GAG concentrations. J Biomed Mater Res A 91, 92-101.

Weisgerber, D.W., Caliari, S.R., Harley, B.A., 2015a. Mineralized collagen scaffolds induce hMSC osteogenesis and matrix remodeling. Biomater Sci 3, 533-542.

Weisgerber, D.W., Caliari, S.R., Harley, B.A.C., 2015b. Mineralized collagen scaffolds induce hMSC osteogenesis and matrix remodeling. Biomater Sci 3, 533-542.

Weisgerber, D.W., Kelkhoff, D.O., Caliari, S.R., Harley, B.A., 2013. The impact of discrete compartments of a multi-compartment collagen-GAG scaffold on overall construct biophysical properties. J Mech Behav Biomed Mater 28, 26-36.

Wettergreen, M.A., Bucklen, B.S., Sun, W., Liebschner, M.A., 2005. Computer-aided tissue engineering of a human vertebral body. Ann Biomed Eng 33, 1333-1343.

Williams, J.M., Adewunmi, A., Schek, R.M., Flanagan, C.L., Krebsbach, P.H., Feinberg, S.E., Hollister, S.J., Das, S., 2005. Bone tissue engineering using polycaprolactone scaffolds fabricated via selective laser sintering. Biomaterials 26, 4817-4827.

Woodard, J.R., Hilldore, A.J., Lan, S.K., Park, C.J., Morgan, A.W., Eurell, J.A., Clark, S.G., Wheeler, M.B., Jamison, R.D., Wagoner Johnson, A.J., 2007. The mechanical properties and osteoconductivity of hydroxyapatite bone scaffolds with multi-scale porosity. Biomaterials 28, 45-54.

Xie, J., Peng, C., Zhao, Q., Wang, X., Yuan, H., Yang, L., Li, K., Lou, X., Zhang, Y., 2015. Osteogenic differentiation and bone regeneration of the iPSC-MSCs supported by a biomimetic nanofibrous scaffold. Acta Biomater.

Zhang, H., Migneco, F., Lin, C.Y., Hollister, S.J., 2010. Chemically-conjugated bone morphogenetic protein-2 on three-dimensional polycaprolactone scaffolds stimulates osteogenic activity in bone marrow stromal cells. Tissue Eng Part A 16, 3441-3448.

Zopf, D.A., Mitsak, A.G., Flanagan, C.L., Wheeler, M., Green, G.E., Hollister, S.J., 2014. Computer aided-designed, 3-dimensionally printed porous tissue bioscaffolds for craniofacial soft tissue reconstruction. Otolaryngol Head Neck Surg. 


\section{Figures}

Figure 1

(a)
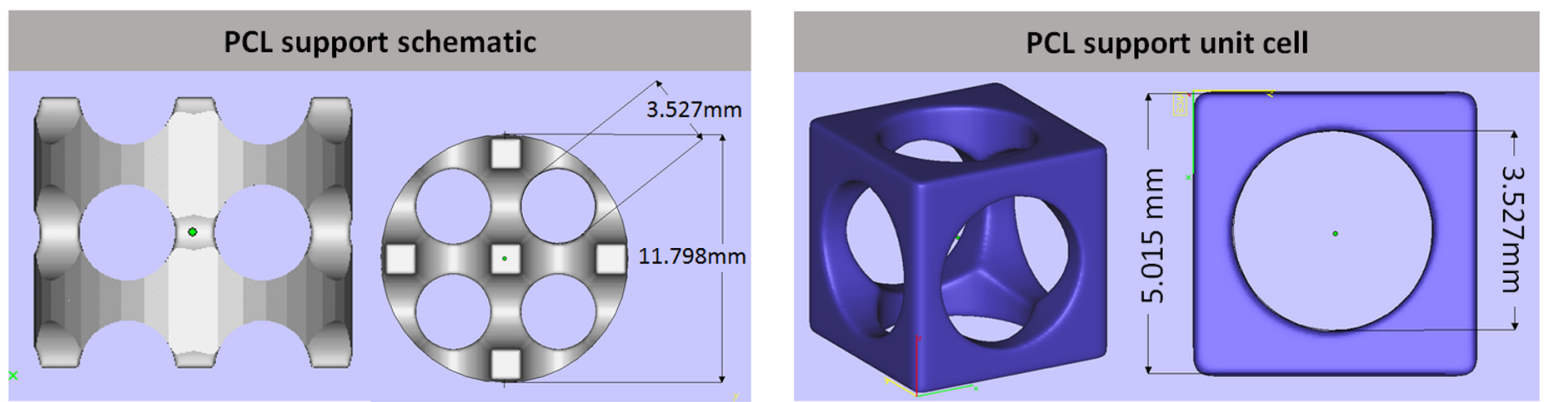

(b)

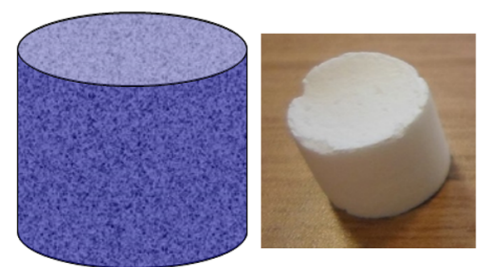

CGCaP Scaffold

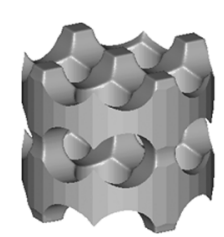

PCL Support
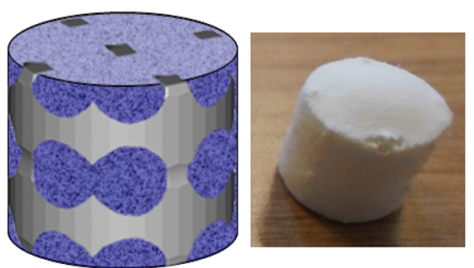

CGCaP-PCL Composite

(c)
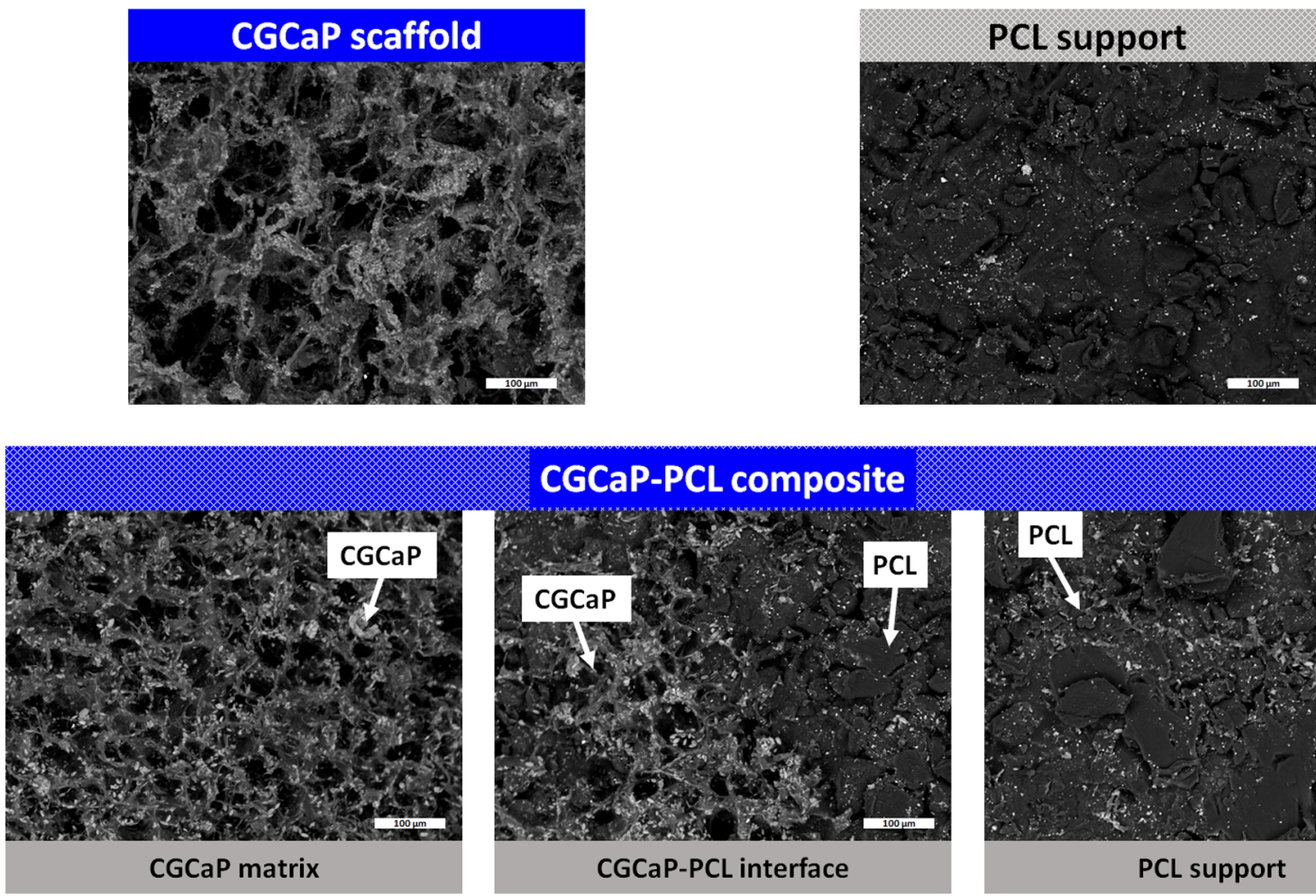

\section{CGCaP-PCL composite}
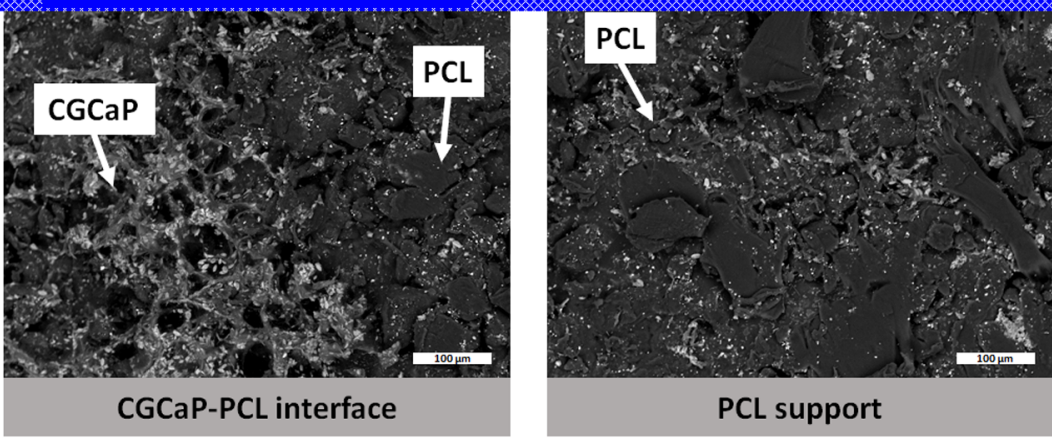
Figure 2

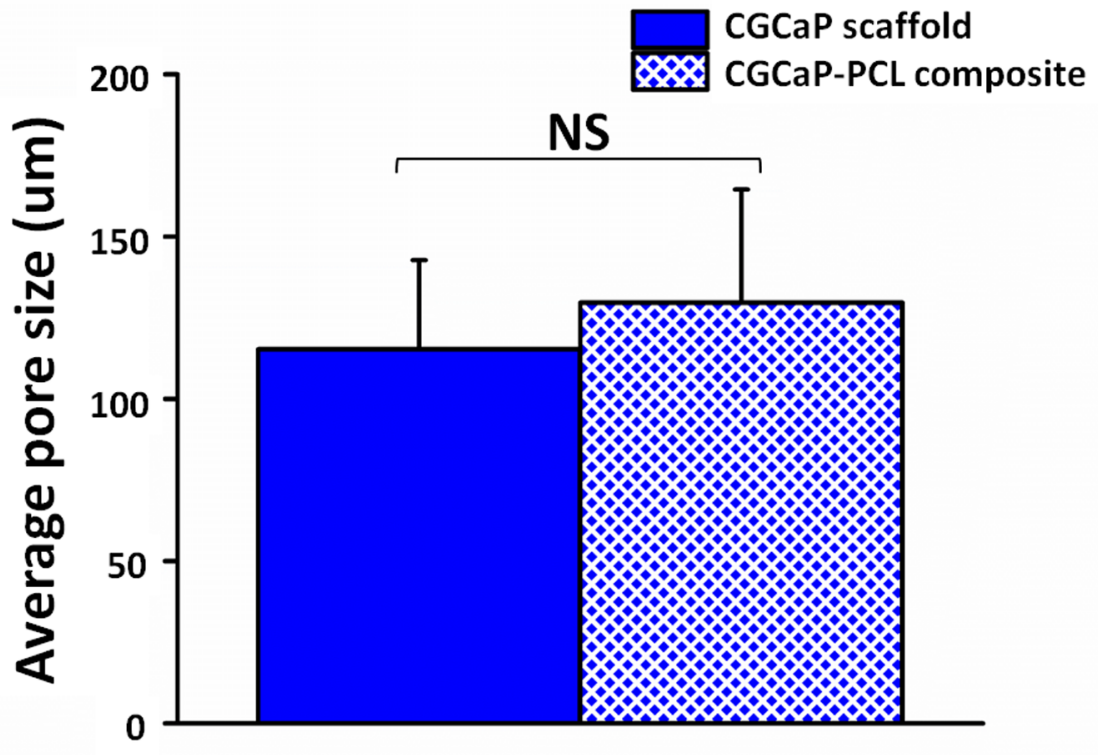


Figure 3
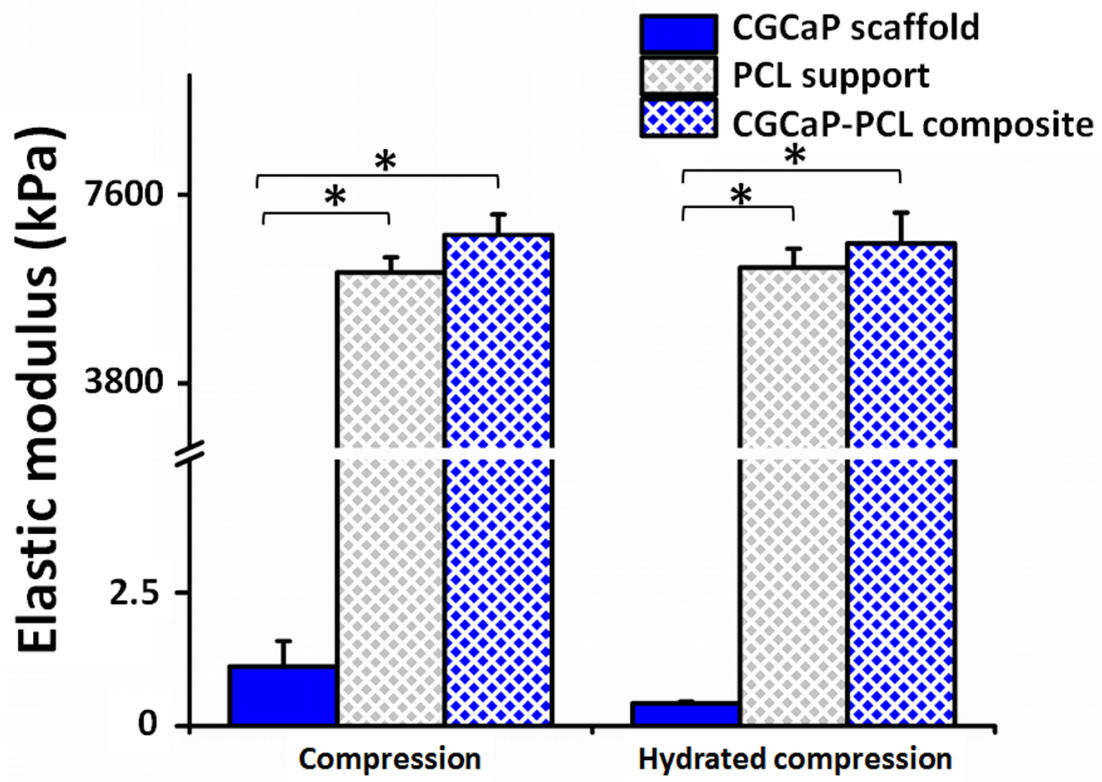
Figure 4

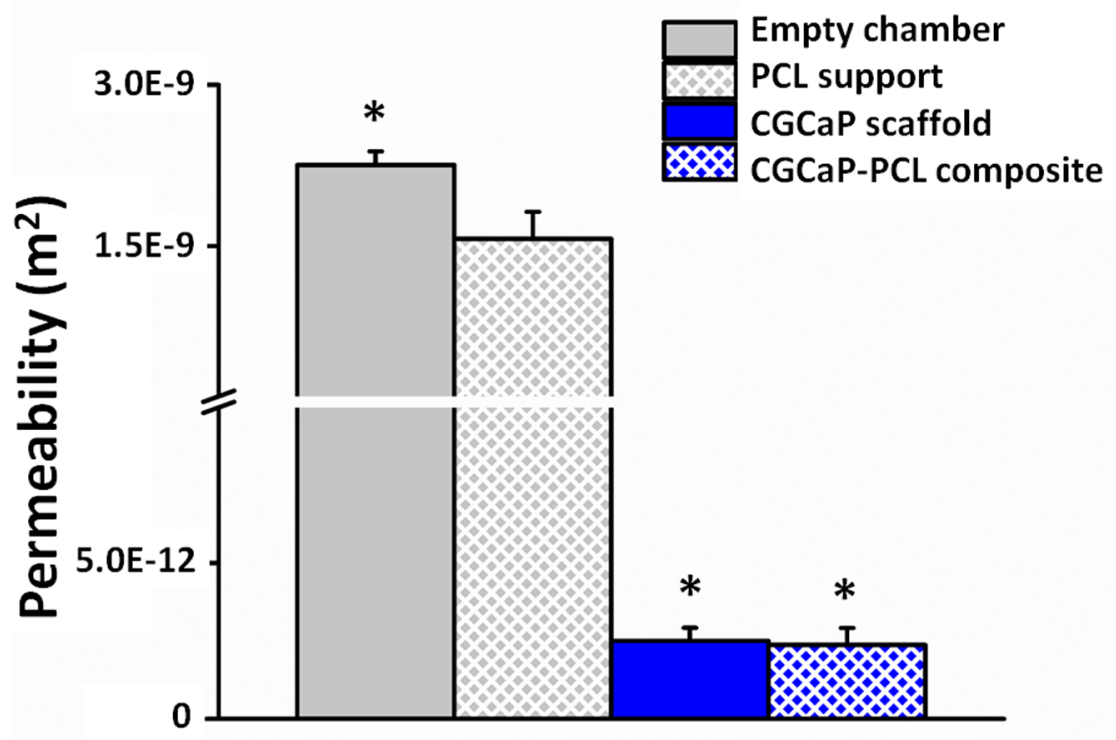

Empty chamber PCL support CGCaP scaffold CGCaP-PCL composite 


\section{Figure 5}

A

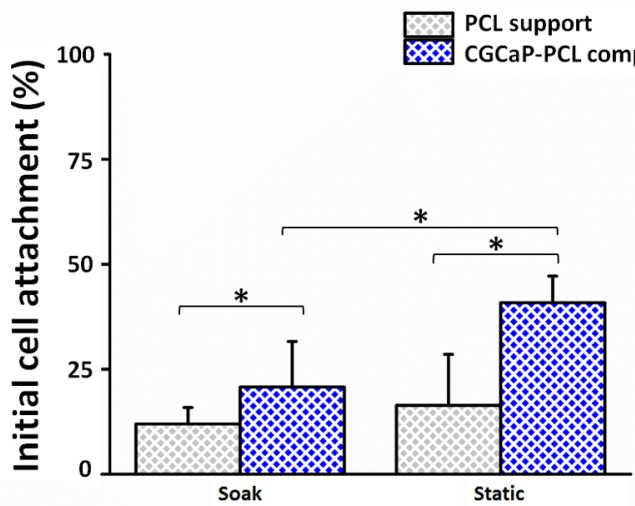

B

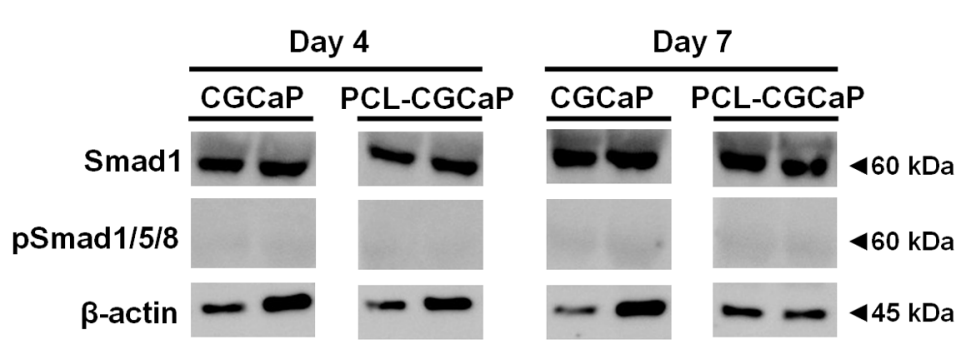


Collagen-GAG scaffold
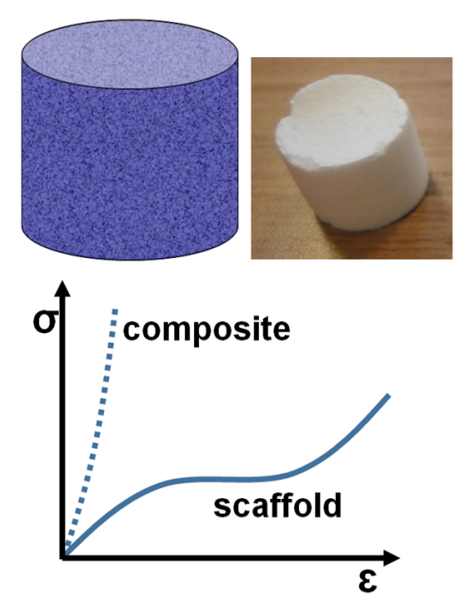

Bulk mechanics
Polycaprolactone (PCL) construct
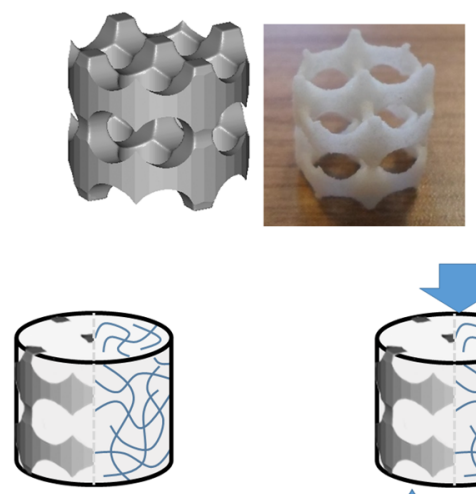

Specific surface area

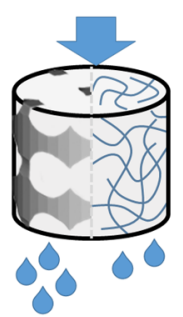

Permeability
PCL-collagen composite
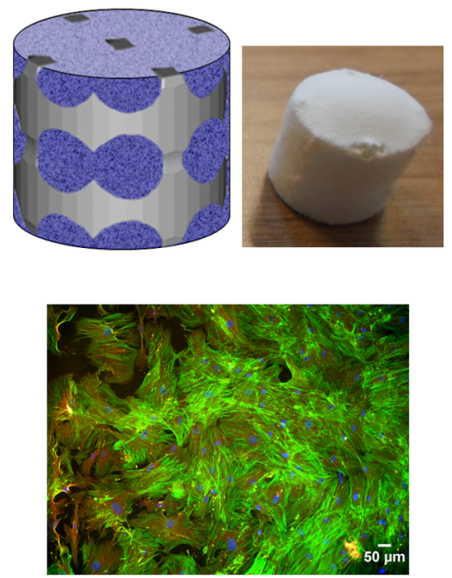

Cell attachment 